\title{
Virtual reality exposure therapy for adolescents with fear of public speaking: a non-randomized feasibility and pilot study
}

Smiti Kahlon ${ }^{1 *} \mathbb{D}$, Philip Lindner ${ }^{1,2,3}$ and Tine Nordgreen ${ }^{1,4}$

\begin{abstract}
Background: Public Speaking Anxiety (PSA) is a common anxiety with onset in adolescence and early adulthood. With the advent of consumer virtual reality (VR) technology, VR-delivered exposure therapy is now a scalable and practical treatment option and has previously been shown to be efficacious with adults. In this non-randomized feasibility and pilot trial, we explore the effect of one-session (90 $\mathrm{min}$ ) VR-delivered exposure therapy for adolescents (aged 13-16) with PSA.
\end{abstract}

Methods: A total of 27 adolescents were recruited from Norwegian high schools and completed self-report measures of PSA twice prior to treatment, 1 week after treatment, and at 1 and 3 month follow-up. Heart rate was recorded during the treatment session. A low-cost head-mounted VR display with a custom-built VR stimuli material depicting a cultural and age appropriate classroom and audience were used when a series of speech (exposure exercises) were performed.

Results: Linear mixed effects model revealed a significant decrease in PSA symptoms (Cohen's $d=1.53$ ) pre-post treatment, and improvements were maintained at follow-ups. Physiological data revealed a small increase in heart rate during exposure tasks. Based on feedback from the adolescents, the feasibility of the intervention was increased during the trial.

Conclusions: The results show that low-cost, consumer VR hardware can be used to deliver efficacious treatment for PSA in adolescents, in a feasible one-session format.

Keywords: Virtual reality, Virtual reality exposure therapy, Public Speaking Anxiety, Cognitive behavior therapy, Adolescents, Inhibitory learning

\section{Background}

One out of three report anxiety symptoms when giving a speech in front of others [1], referred to as Public Speaking Anxiety (PSA). The most common fears in performance situations among individuals with PSA include showing signs of anxiety symptoms like shaking or trembling, the mind going blank while presenting,

*Correspondence: smiti.kahlon@helse-bergen.no

${ }^{1}$ Division of Psychiatry, Haukeland University Hospital, Haukelandsbakken 15, 5009 Bergen, Norway

Full list of author information is available at the end of the article saying something stupid or not being able to continue to talk [2]. PSA is a distinct subgroup of the wider clinical presentation of social anxiety disorder (SAD) [3]. SAD is defined as the fear of negative evaluation of others in social situations, followed by feeling embarrassed or humiliated [4]. SAD is one of the most common psychiatric disorders with a life time prevalence of $13.7 \%$ in the Norwegian general population [5] and $4.0 \%$ across all countries [6]. The majority of the individuals with SAD report anxiety in performance situations [2]. SAD has an onset in adolescence with a mean age onset at 15 years [2, 7]. Over $80 \%$ of the individuals with SAD do not receive

(c) The Author(s) 2019. This article is licensed under a Creative Commons Attribution 4.0 International License, which permits use, sharing, adaptation, distribution and reproduction in any medium or format, as long as you give appropriate credit to the original author(s) and the source, provide a link to the Creative Commons licence, and indicate if changes were made. The images or other third party material in this article are included in the article's Creative Commons licence, unless indicated otherwise in a credit line to the material. If material is not included in the article's Creative Commons licence and your intended use is not permitted by statutory regulation or exceeds the permitted use, you will need to obtain permission directly from the copyright holder. To view a copy of this licence, visit http://creativecommons.org/licenses/by/4.0/. The Creative Commons Public Domain Dedication waiver (http://creativecommons.org/publicdomain/zero/1.0/) applies to the data made available in this article, unless otherwise stated in a credit line to the data. 
any treatment, and the mean age of first treatment is at 27 years [2].

The literature describes two subgroups of adult SAD: those with both interaction and performance anxiety (generalized SAD) and those with only performance anxiety [3], with PSA as the most common symptom in both adolescents [8] and adults [9]. Congruently, the novel DSM-5 [4] revised its specifier of SAD to include a "performance only" subgroup, distinct in terms of etiology, age at onset, physiological response, and treatment response [10].

Untreated PSA may cause further impairment in adulthood and around 50\% of adolescents [11] and adults [3] with PSA develop generalized social anxiety [7]. Studies have shown that treating the specific subtype of PSA reduce the overall level of generalized social anxiety [1214] and by providing a treatment intervention targeting adolescents, one might be able to reduce the societal and personal costs of the disease.

There is strong evidence supporting cognitive behavioral therapy (CBT) with exposure as the treatment of choice in treating PSA and SAD $[15,16]$. However, conducting in-session exposure exercises for PSA has historically been unpractical or outright infeasible since this would require access to and control over an audience. This is in contrast with treatments of other anxiety disorders that rely on in-session exposure therapy, e.g. animal phobias and other specific phobias that are highly efficacious [17-20]. Virtual reality (VR) technology can resolve this issue by creating the illusion of being present in front of a realistic virtual audience. This is achieved by wearing a headset with dual displays that cover the eyes and simulates depth perception, the displayed content of which is interactive to head movement to give the illusion of being able to look around the virtual world [21]. By creating an animated virtual audience and presenting the feared stimuli to the patient, VR Exposure Therapy (VRET) for PSA is an attractive treatment method since it provides a convenient way doing in-session exposure with immediate access to controllable fear stimuli. Importantly, virtual audiences are sufficient to elicit a fear response [22], the basis of exposure therapy, and several randomized controlled trials of VRET for PSA have shown good results [23-26].

Until recently however, VR equipment was expensive, inaccessible, and required a high degree of technical competence to develop for and use. Lindner et al. [26] was the first to investigate whether consumer VR hardware and software can be used to conduct in-session exposure therapy with a therapist. One study has showed that relevant VR-stimuli do provoke distress in socially anxious youth [27]. To our knowledge, there have been no intervention studies on VRET for adolescents with
PSA. In the current, non-randomized feasibility and pilot study, we investigate the feasibility of adapting the 3-h single session VRET protocol for PSA examined in Lindner et al. [26] into a 90-min single session for use with adolescents, and examine whether the effect size is similar, explore possible moderators of treatment effects, and physiological response to the VR scenarios.

\section{Methods \\ Design}

This study is a non-randomized feasibility and pilot study with pre, post and 1- and 3-month follow-ups. Reporting follows the CONSORT guidelines for pilot/feasibility trials.

\section{Ethics}

The study received ethical approval from the Norwegian Regional Ethical Committee (REK 2017-1521). Written informed consent was obtained from the parents of adolescents at the training session.

\section{Procedure}

A total of $N=27$ adolescents were included in the feasibility and pilot study. Recruitment was done in two periods: spring 2018 and autumn 2018. Information about the study was given in classrooms at two high schools (8th to 10th grade). In addition, written information with a link to the study website was distributed to all students and parents through mail, as well as school health services and head masters at the schools in Bergen, Norway, and through Facebook. Interested participants accessed the study website and completed the online screening, including Public Speaking Anxiety Scale (PSAS; [28]) and Social Interaction Anxiety Scale (SIAS; [29]). In order to be included, the adolescents had to be between 13 and 16 years old, confirm that they were afraid of speaking in public, had to report symptoms of PSA on the PSAS (observed range: 46-73, possible range 17-85) and functional impairment due to PSA. Due to the lack of international established cut-offs, no threshold level for PSAS was applied. Exclusion criteria, assessed during the same phone call, were: ongoing psychotherapy, use of benzodiazepines, and lack of stereoscopic vision that would impair the VR experience. After the initial screening eligible participants were contacted by phone to schedule a date for the training session.

Participants met for a single 90-min training session approximately 1 week after completing the online pretreatment questionnaires. Informed consent from parents was obtained before the exposure session. Posttreatment questionnaires were distributed 1 week after the training session. In addition, follow-up questionnaires were distributed one and 3 months after completing the 
treatment session, in order to evaluate the long-term effects of the treatment. See Fig. 1 for study flowchart. Participants who completed the training session and responded to the questionnaires received a gift certificate of 200 NOK (approximately 20 Euros) when completing the 1-month follow-up assessment.

\section{Intervention}

\section{VR scenario}

A custom-built VR stimuli material depicting a typical Norwegian classroom and an age-appropriate audience was developed by Attensi AS, a professional IT developer. The scenario was inspired by five culturally and age-appropriate actual Norwegian classrooms and adjusted after feedback from testing by four adolescents. The classroom featured ten virtual avatars, depicted to be in the age range of $13-16$, sitting at their desks, with

Day 0: Screening $(n=38)$

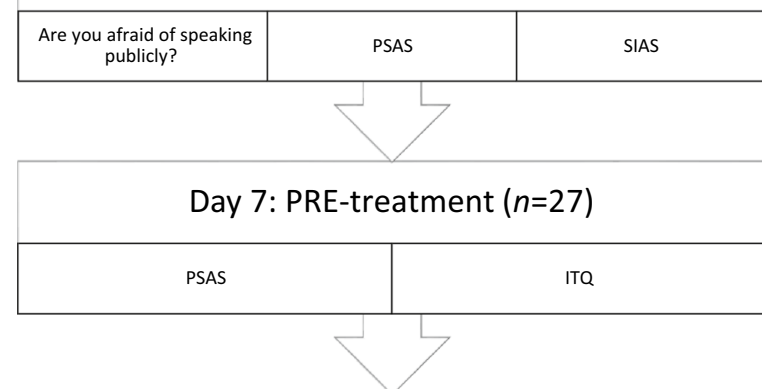

Day 14: 90 minutes single session $(n=27)$



Fig. 1 Study flowchart minor body animations and gaze directed at the user situated at the front of the class. An empty classroom and a lobby were also available, with each exposure exercise beginning with the participant selecting to enter the full classroom. See Fig. 2 for screenshots. An Apple iPhone 7 and a high-end Cardboard-type VR headset (costing the equivalent of 60 USD) was used for stimuli presentation. The application automatically logged all user behaviors (e.g. entering and exiting the full classroom) with timestamps.

\section{Treatment protocol}

The treatment protocol was adapted from a recent VRET protocol for PSA [26], and tailored for use with adolescents. This protocol in turn was based on Öst's [20] one-session treatment for specific phobias, with speech (exposure) exercises (e.g. count down from 60, say words beginning with the letter $\mathrm{P}$, improvised speeches about everyday matters), partly drawn from a one-session in vivo exposure protocol for PSA [12]. The session was therapist-led and consisted of three parts: brief psychoeducation (approx. $15 \mathrm{~min}$ ), followed by exposure tasks (approx. $60 \mathrm{~min}$ ) and ending with a summary and introduction to active maintenance (approx. $15 \mathrm{~min}$ ). The therapeutic goal was to promote inhibitory learning [30] by exposing participants to speech scenarios designed to test their idiosyncratic catastrophic beliefs about speech performance and physiological reaction. The psychoeducation consisted of a short introduction to the CBT model of anxiety with some VR-adjustments. A simple functional analysis of their PSA was performed and the treatment rationale was explained.

The treatment protocol included seven tasks with varying levels of difficulty that lasted 1 to 2 min each with no or little preparation time. Adolescents wore the VR device only during the actual exposure tasks in order to avoid habituation to the virtual environment in itself, outside an exposure context. Participants were first instructed to enter a neutral empty classroom to make sure that the VR device was correctly configured. The adolescents were then exposed to a virtual classroom with an animated audience. Each task required entering and leaving the virtual classroom from a lobby, with discussions with the therapist between the tasks. Prior to each exposure task, the therapist would give instructions and noting their catastrophic beliefs and expectancy rating on a scale from 0 to 100 . The therapist recorded maximum and minimum subjective units of distress (SUD; [31]) on a $0-100$ scale, where 0 corresponds to no distress at all and 100 is the worst possible, as reported by the participant directly after each exposure task. SUDS [31] is a useful tool by showing the participants how their level of anxiety reduces throughout the exposure 


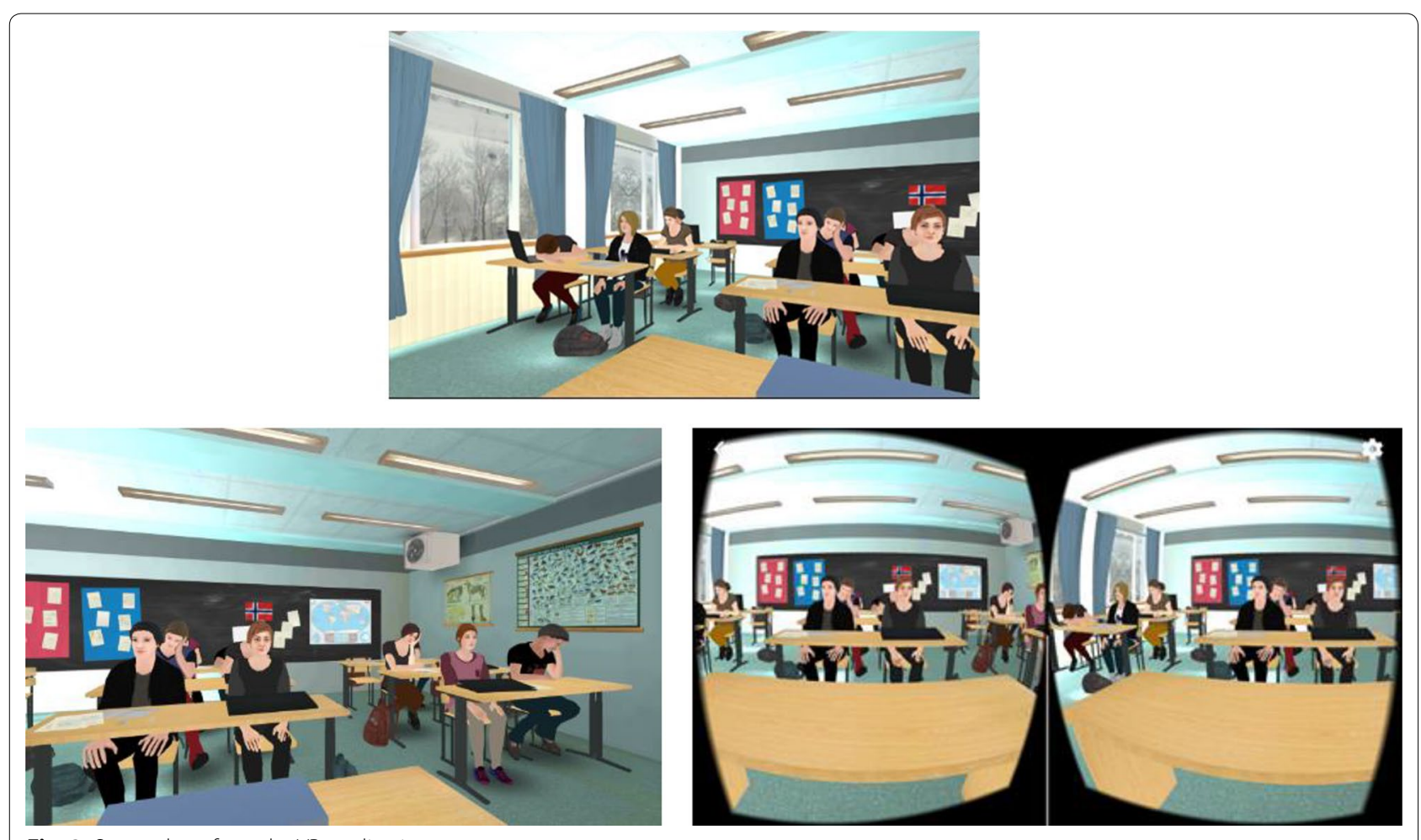

Fig. 2 Screenshots from the VR application

tasks. Immediately after the exposure task, the adolescent would evaluate the performance together with the therapist and rate the subjective level of discomfort experienced. The therapist and adolescent then listened to an audio recording of the task, with the adolescent using mental imagery to cast themselves in the role of an audience member in the same scenario listening to the speech as played back. Participants were then asked to once again quality rate the performance after listening and discuss with the therapist about discrepancies before continuing to the next exposure task. This procedure was then repeated for each task.

After completing the exposure tasks, the adolescent and the therapist had a short summary discussion before introducing them how to practice exposure in real life. Main points were summed up in a folder, which was given to the adolescent at the end of the session. The duration of the session was reduced from an original $3 \mathrm{~h}$ [26] to approximately $90 \mathrm{~min}$, thus making the session more age appropriate. All training sessions were conducted at a hospital location after school hours. In order to ensure further in vivo exposure, the participants were contacted by telephone 2 weeks after the training session. A short assessment was then made of the participant's experience in participating in the session. In addition, the participants were given prompts for data purposes.

\section{Therapists}

The sessions were conducted by two clinical psychologists with experience in CBT treatments of social anxiety disorder and received $4 \mathrm{~h}$ of protocol-specific training. The same therapist that conducted the session also contacted the participants by telephone after the training session. The therapists were supervised by a senior clinical psychologist throughout the treatment period.

\section{Measurements \\ Primary outcome measure}

The Public Speaking Anxiety Scale (PSAS; [28]) was administered at screening, at the beginning of the session, post-treatment and at 1 and 3-month follow-up. The PSAS covers cognitions, behaviors and physiological manifestations of Public Speaking Anxiety with 17-items, with a five-point Likert response format. Five of the items are reverse coded. The questionnaire was translated into Norwegian for this study according to scientific standard (including back-translation). Cronbach's alpha at pretreatment was an acceptable 0.76 .

\section{Moderators of treatment effect}

Two possible moderators of treatment effects were examined. The Social Interaction Anxiety Scale (SIAS; [29]) was administered at screening and measures the more 
generalized type of SAD which includes interaction anxiety. SIAS is a 20 -items scale on a scale from 1 "not at all characteristic or true of me" to 4 "extremely characteristic or true of me". Summary scores ranges from 20 to 80 with a higher score indicating more social interaction anxiety. SIAS show good psychometric properties, and discriminates patients with social phobia from patients with other anxiety disorders or no disorder at all. Cronbach's alpha at screening was calculated to 0.86 . As the literature distinguishes between the two subgroups of SAD [3], we investigated whether performance-only social anxiety had better treatment outcome than adolescents with the more generalized type of social anxiety, which includes both performance and interaction anxiety. A binary variable corresponding to "low SIAS" (0, reference) and "high SIAS" (1) was created using mediansplit subsampling (median $=35$ ), creating two groups $\left(n_{\text {low }}=\geq 35, n_{\text {high }}=\leq 36-100\right)$. This binary variable was used to examine moderating effects, since interaction effects were unlikely to be linear.

Sense of presence in VR environment, the degree to which the experience feels real [32], was also explored as a possible moderator of treatment effects. Sense of presence is positively associated with emotional distress in VR [33], yet the causal, possibly bi-directional relationship between presence and emotional distress is complex [34]. In this study, the Gatineau Presence Questionnaire [35] was administered at the end of the session. The Gatineau Presence Questionnaire is a short measure with five items rated on a $0-100$ scale resulting in an average score in percentage. The questionnaire assesses (1) the impression of being in the virtual environment, (2) the experience as being real, and the reversed items; (3) attentiveness of the virtual environment as being artificial, (4) the experience of being present in the office instead of the virtual environment and (5) the experience of discomfort. A presence score is calculated by averaging items $1-4$. A binary variable "low presence" (0, reference) and "high presence" (1) was then created using mediansplit subsampling, creating two groups $\left(\mathrm{n}_{\text {low }}=\geq 59\right.$, $\mathrm{n}_{\text {high }}=\leq 60$. This binary variable was used to examine moderating effects, since interaction effects were unlikely to be linear.

\section{Physiological data}

Heart rate data was collected continuously during the session using a wearable, wireless Empatica E4 wristband. Timestamped heart rate data were synchronized to the log files of the VR-running smartphone such that for each data point (temporal resolution: $1 \mathrm{~s}$ ), the virtual scenario in which each data point was recorded was known. In total, $n=147,322$ data points from $n=21$ participants were available for analyses. Time spent in the lobby before entering the full classroom served as comparison period for each exposure period, with the first $60 \mathrm{~s}$ of data after each exposure period being discarded to allow heart rate normalization. Mean heart rate during each period and task were calculated. The median number of recorded transitions from lobby to full classroom was eight, with a maximum of 14 . Since repetitions of and deviations from the seven per-protocol speech exercises was not systematically recorded, it is not possible to assert whether recoded exposure tasks are equivalent across participants. Only the initial eight exercises for each participant were included in analyses since these likely show the least variations across participants.

\section{Analyses}

SPSS Statistics version 24 was used to analyze data. Outcome data were analyzed using linear mixed effects models [36], modeling change on both individual and group level. The analysis included unstructured random effects covariance matrices, random slopes and intercept. All participants who began treatment were included in analyses, with missing data estimated using restricted maximum likelihood modeling of random effects. PSAS scores served as the dependent variable in all models, with a binary independent variable corresponding to before and after treatment (both screening and pre-measurement coded as zero and the post-measurement as one). Moderation analyses were performed using the same time variable, a binary moderator, and the interaction thereof. In the analysis of long-term effects, a separate mixed model was run using a new, numeric time variable corresponding to months since treatment $(0=$ post, $1=1$-month follow-up, 3=3-months follow-up). Calculation of effect sizes was based on estimated means pre and post treatment: $\operatorname{Pre}_{\mathrm{m}}-$ Post $_{\mathrm{m}} / \mathrm{SD}_{\text {pre }}$ where the calculation of standard deviation $=$ standard error $\times \sqrt{ } \mathrm{N}$. Heart rate data were also analyzed using mixed models (random slopes and intercepts), with period-average heart rate as the dependent variable and period (lobby or full classroom) as independent variable.

\section{Results}

\section{Attrition}

A total of 38 participants completed the online screening, 32 participants $(84.2 \%)$ were invited to participate in the study and to the training. Two participants cancelled the training session due to lack of time, one did not show up at the training session, one participant was referred to other health services and one participant was excluded due to missing consent from parents. This resulted in $N=27$ participants: $n=6$ male (22\%) and $n=21$ female (78\%). Participants ranged from 13 to 16 years old, with an average age of 14.22 years $(S D=0.64)$. Observed mean 
Table 1 Observed means, standard deviations and $n$ missing for primary outcome measure (PSAS) at each measure point

\begin{tabular}{lllll}
\hline Day & Assessment & $\boldsymbol{M}$ & $\boldsymbol{S D}$ & $\boldsymbol{n}$ missing \\
\hline 0 & Screening & 62.81 & 9.88 & 0 \\
7 & Pre-treatment & 61.04 & 7.80 & 0 \\
21 & Post-treatment & 49.28 & 10.23 & 2 \\
42 & One-month follow-up & 50.00 & 12.73 & 1 \\
97 & Three-months follow-up & 47.25 & 11.92 & 3 \\
\hline
\end{tabular}

$M$ mean, SD standard deviation, $n$ : number of participants, PSAS Public Speaking Anxiety scale

pretreatment level and the mean change and standard deviations for the primary outcome variable PSAS are presented in Table 1.

\section{Changes from pre-treatment to post-treatment}

Table 1 shows observed means and standard deviations for all measure points. PSAS symptoms remained stable from screening to pre-treatment. The unconditional mixed model showed a significant decrease in their PSAS score from pre to post by an average of 12.23 points $(S E=2.08, p<0.001)$. The effect size, calculated from estimated means pre and post treatment and standard error: $61.04-48.81 / 7.98=1.53$.

\section{Changes from Post-treatment to Follow-ups}

Modeling the follow-up period revealed a non-significant decrease in PSAS score of $-0.44(S E=0.41, p=0.300)$ per month after treatment. See Table 1 for observed scores.

\section{Moderators of treatment effects}

Mixed effects models were computed in order to investigate whether there was any difference between groups when exploring moderators of treatment effects, by using low generalized social anxiety and low experience of presence as a reference in the analyses. Moderators of treatment effects showed no difference between groups in treatment outcome from pre to post. See Table 2 for details.

\section{Physiological response to exposure}

Average heart rate during exposure was $85.89(S E=1.59)$, rising on average $3.66(S E=1.03, p<0.001)$ from the nonexposure period immediately preceding it. Plotting the data over time revealed that this difference differed substantially between tasks, but because task-equivalence across participants cannot be assumed, it is not possible to draw conclusions as to whether some tasks led to greater increases of heart rate. See Fig. 3.
Table 2 Estimated treatment effects on primary outcome measures (PSAS)

\begin{tabular}{|c|c|c|c|c|}
\hline & $\beta$ & $S E$ & $p$ & $95 \% \mathrm{Cl}$ \\
\hline \multicolumn{5}{|c|}{ Unconditional pre-post } \\
\hline (Intercept) & 61.93 & 1.56 & $<0.001$ & 58.72 to 65.13 \\
\hline Time & -12.23 & 2.08 & $<0.001$ & -16.52 to 7.94 \\
\hline \multicolumn{5}{|c|}{ Moderation pre-post by presence (median-split) } \\
\hline (Intercept) & 60.36 & 2.16 & $<0.001$ & 55.91 to 64.81 \\
\hline Presence & 2.48 & 3.18 & 0.443 & -4.08 to 9.03 \\
\hline Time & -10.99 & 3.19 & $<0.01$ & -17.57 to 4.40 \\
\hline Presence $\times$ time & -4.04 & 4.60 & 0.389 & -13.54 to 5.46 \\
\hline \multicolumn{5}{|c|}{ Moderation pre-post by generalized SAD (median-split) } \\
\hline (Intercept) & 60.04 & 2.12 & $<0.001$ & 55.67 to 64.40 \\
\hline SIAS & 3.93 & 3.05 & 0.21 & -2.36 to 10.21 \\
\hline Time & -14.68 & 2.82 & $<0.01$ & -20.51 to -8.85 \\
\hline SIAS $\times$ time & 5.10 & 4.07 & 0.22 & -3.31 to 13.51 \\
\hline
\end{tabular}

$N=27$

$B$ (unstandardized) parameter estimates, $S E$ standard error, $\mathrm{Cl}$ confidence interval

\section{Feasibility outcomes}

One main purpose of this study was to examine the feasibility of the protocol. Five elements were adjusted from the first (spring 2018) to the second period (autumn 2018) of the study.

\section{Parent involvement}

Parents were more involved in the second period compared to the first period through e-mail distribution and telephone contact. They were given more detailed information at the beginning of the exposure session, and were asked to ensure that the adolescents fill out the follow- up assessments.

\section{Attrition}

During the first period, the gift card was distributed to the adolescents after completion of 1-month follow-up, which resulted in difficulties in collecting the 3-month follow-up questionnaire. By adjusting the value of the gift card and distributing them to the adolescents after having completed the 3-month questionnaires, the study did not have any missing data during the second period.

\section{Compensation}

Due to the workload for the adolescents, we increased the value of the gift card from 200 to 300 NOK (30 euros). 


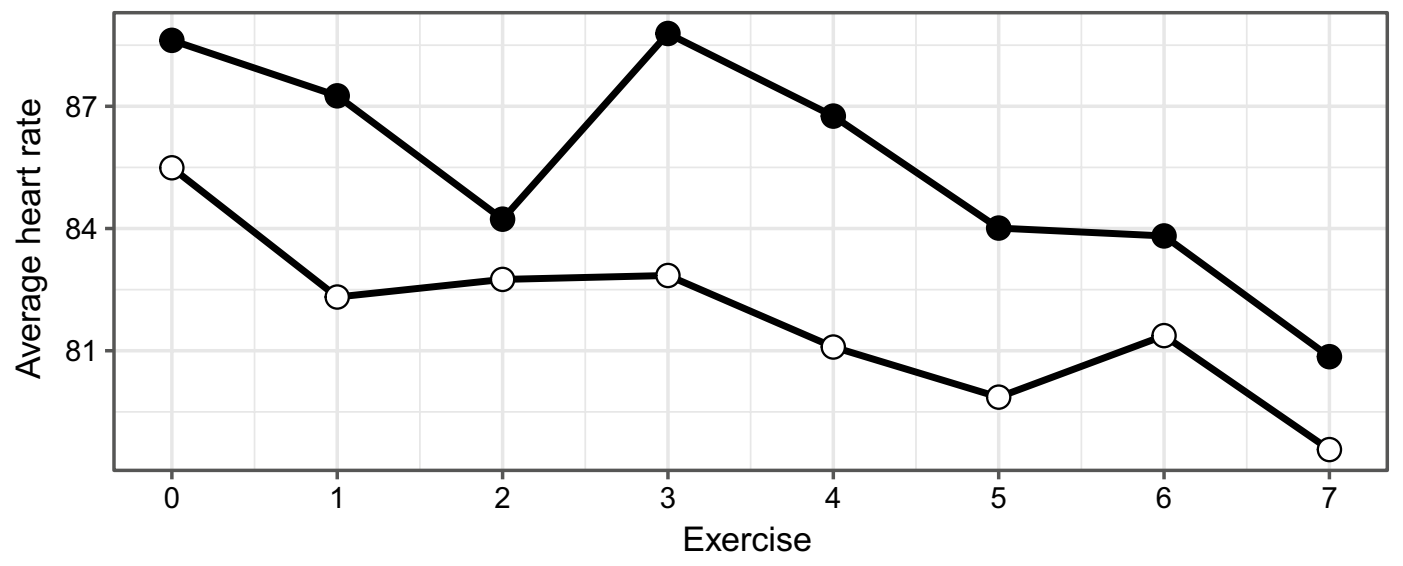

During exposure $\bigcirc$ In between exposure

Fig. 3 Heart rate

\section{Break}

Due to the long duration after school hours, a short break was included halfway through the intervention and before starting on the relapse prevention part.

\section{Duration of training session}

According to the protocol, the intervention was supposed to be $90 \mathrm{~min}$. However, this was not applicable and time spent on each training session was between 2 and $2.5 \mathrm{~h}$.

\section{Discussion}

The present study is the first to examine the feasibility, to pilot the effects of a VRET intervention for adolescents with fear of public speaking. In addition, the aim was to examine moderators of treatment and heart rate during the intervention.

A total of $N=27$ eligible adolescents between 13 and 16 years participated in the study, the majority being female. The original protocol was developed for adults, and adjusted for this age group. Through feedback from the participating adolescents and the therapists, a further adaption to the target group was conducted during the study period. This included increasing the training session from 90 to $120-150 \mathrm{~min}$ with a short break, increasing parent involvement, increasing gift card value and introducing prompts after the treatment session for in vivo exposure and data-collection purposes.

The one-session treatment showed large effects on the primary outcome measure (PSAS, ES =1.53) 1 week after treatment. This result remained stable during the one and three follow-up period. This result is comparable to the findings from previous studies on VRET for PSA for adults [23, 25, 26, 37]. The present study showed a large effect size and a reduction in PSAS scores of 12.23 points, whereas the Lindner et al. [26] study showed a 6.90 point reduction. This difference indicate that the original protocol was successfully adapted before and during the trial to our target group. This includes the development of a VR stimuli tailored specifically to illustrate a cultural and age appropriate classroom and audience.

With regards to treatment moderators, the results revealed no significant differences in symptom reduction on the basis of a high general social anxiety symptoms (SIAS scores). Neither did our results show a moderating effect of high sense of presence. While previous research has shown robust associations between presence and emotional distress during the VR exposure, the role of presence in explaining treatment outcome remains unclear $[34,38]$. Of note, both these moderation analyses were low-powered, and should therefore be interpreted with caution.

Results from analyses on physiological data revealed that the exposure scenarios were successful in eliciting a physiological response in the form of increase heart rate, yet the increase was small. This is in line with clinical experience from the Lindner et al. [26] trial, finding that VR public speaking scenarios elicit a weaker fear response compared to e.g. VR spider scenarios [39]. The combination of a relative weak physiological response during treatment and a large decrease in the psychological symptoms is congruent with the theoretical foundation for the treatment protocol, emphasizing inhibitory learning over a strong fear response and subsequent habituation rationale [30]. This is 
also consistent with empirical findings showing that a strong fear response is not necessary for fear extinction [40].

PSA has been until now difficult to treat in a traditional therapeutic setting, due to the stimuli required. VRET is not a common method in the healthcare services, as VRdevices has not been easily available, and has a high cost. With innovative and accessible consumer technology, VRET for PSA may now be offered as a tool for therapists, and VRET can be easily conducted in any clinical setting. The therapists involved in the feasibility and pilot study had a CBT background with no prior experience with VRET, and were able to conduct the treatment after only a 4-h workshop. This shows how any CBT clinician can use the treatment method after only a small amount of training. Also, studies conducted both before and after the advent of available consumer VR technology, have shown that clinicians see benefits of using VR to conduct exposure therapy and that they are willing to adopt the technology in clinical practice $[26,41,42]$.

Importantly, we also replicate the finding that modern, low-cost consumer VR hardware can be used to administer efficacious treatment [26]. Findings from this study shows how low-cost VR can be used in treatment and by using a mobile app the potential for the scalability of the treatment method.

\section{Strengths and limitations}

One major strength of this study is low attrition among adolescents as the study had few missing data. Another major strength of this study is the investigation of physiological data using heart rate as well as collecting the PSAS symptom scores, which gives valuable information on both their subjective and their objective level of discomfort experienced when talking to an audience.

There are also some limitations of this study, which needs to be addressed. No control or comparison groups were included in this feasibility and pilot study. Consequently, we cannot conclude on cause for change during the intervention. For example, a decrease in PSA symptoms can be attributed to less oral presentations at school during the treatment and follow-up period. Moreover, the current design cannot isolate the therapist effect from the VRET effect, as the participants conducted the VRET intervention with guidance from a therapist. Being adjacent to a therapist when doing the exposure tasks might have contributed to the clinical efficacy of the VR intervention. However, the recent randomized controlled trial conducted by Lindner et al. [26] showed a reduction in anxiety symptoms when doing the intervention at home without therapist-guidance. Miloff et al. [39] showed that limited therapeutic guidance in VRET for spider phobia led to reduction in anxiety symptoms, and the results maintained the same at follow-up. This indicates that the VR intervention itself is contributing to the reduction in PSA symptoms. Moreover, the study did not collect data on generalized social anxiety disorder (SAD) after treatment; in retrospect, it would have been valuable to include such data at post and follow-up and explore whether VRET for PSA also has a treatment effect on $\mathrm{SAD}$, as indicated by previous studies [12-14]. Another limitation is the small sample (although well-powered for the expected effect size), and as is typical of clinical trials, it is unknown to what degree findings generalize to nontreatment seekers. Further, it consists of a higher group of female.

Future studies should examine the effectiveness of the VR intervention in a randomized controlled trial, with a larger population for the generalizability of the study, in addition to providing the intervention as self-guided for scalability purposes. There is also a need to explore the limitations and benefits of VR-delivered exposure in a head-to-head comparison of in vivo and VR-delivered exposure for FoPS. Data on real-world public speaking behavior during the follow-up intervention would be an interesting outcome measure in future studies, in order to investigate the transition from the VR-scenario to the real world context. In this regard, a longer follow-up period (e.g. 12 months) would have been useful.

\section{Conclusion}

The feasibility and pilot study shows that one-session VRET is an effective tool for treating adolescents with PSA. By using a mobile application platform and an affordable VR platform, the study shows the great potential of VRET as scalable option for treating PSA in adolescents.

\section{Abbreviations}

PSA: Public Speaking Anxiety; SAD: social anxiety disorder; VR: virtual reality; VRET: virtual reality exposure therapy; CBT: cognitive behavioral therapy.

\section{Acknowledgements}

We want to thank all participants and their families. We also want to thank clinical psychologist Marthe Myklebost for conducting half of the training sessions, and Enrique Garcia Ceja for compiling the physiological data.

\section{Authors' contributions}

Designed the study: SK, PL and TN. Treated participants and collected data: SK. Analyzed data: SK, PL and TN. Drafted the manuscript SK. Made significant contributions to writing: PL and TN. All authors read and approved the final manuscript.

\section{Funding}

This project is part of the Introducing Mental health through Adaptive Technology (http://www.intromat.no) funded by the Norwegian Research Council (NFR: 259293)

\section{Availability of data and materials}

The datasets used and/or analyzed during the current study are available from the corresponding author on reasonable request. 


\section{Ethics approval and consent to participate}

The study has received ethics approval from the Regional Ethics Committee (2017/1521). Informed consent was obtained from all participants.

\section{Consent for publication}

Not applicable.

\section{Competing interests}

Author PL reports having received consulting fees from Mimerse. The other authors declare that they have no competing interests.

\section{Author details}

1 Division of Psychiatry, Haukeland University Hospital, Haukelandsbakken 15, 5009 Bergen, Norway. ${ }^{2}$ Department of Psychology, Stockholm University, Stockholm, Sweden. ${ }^{3}$ Centre for Psychiatry Research, Department of Clinical Neuroscience, Karolinska Institutet \& Stockholm Health Care Services, Stockholm County Council, Stockholm, Sweden. ${ }^{4}$ Department of Clinical Psychology, Faculty of Psychology, University of Bergen, Bergen, Norway.

Received: 10 August 2019 Accepted: 17 December 2019

Published online: 27 December 2019

\section{References}

1. Stein MB, Walker JR, Forde DR. Public-speaking fears in a community sample: prevalence, impact on functioning, and diagnostic classification. Arch Gen Psychiatry. 1996:53(2):169-74.

2. Grant BF, Hasin DS, Blanco C, Stinson FS, Chou SP, Goldstein RB, et al. The epidemiology of social anxiety disorder in the United States: results from the national epidemiologic survey on alcohol and related conditions. J Clin Psychiatry. 2005:66(11):1351-61.

3. Blöte AW, Kint MJW, Miers AC, Westenberg PM. The relation between public speaking anxiety and social anxiety: a review. J Anxiety Disord. 2009;23(3):305-13.

4. American Psychiatric Association. Diagnostic and statistical manual of mental disorders. 5th ed. Washington, DC: Author; 2013.

5. Kringlen E, Torgersen S, Cramer V. A Norwegian psychiatric epidemiological study. Am J Psychiatry. 2001;158(7):1091-8.

6. Stein DJ, Lim CCW, Roest AM, de Jonge P, Aguilar-Gaxiola S, Al-Hamzawi $A$, et al. The cross-national epidemiology of social anxiety disorder: data from the World Mental Health Survey Initiative. BMC Med. 2017;15(1):143.

7. Wittchen HU, Fehm L. Epidemiology and natural course of social fears and social phobia. Acta Psychiatr Scand. 2003;108(s417):4-18.

8. Kodal A, Bjelland I, Gjestad R, Wergeland GJ, Havik OE, Heiervang ER, et al. Subtyping social anxiety in youth. J Anxiety Disord. 2017;49:40-7.

9. Furmark T, Tillfors M, Everz PO, Marteinsdottir I, Gefvert O, Fredrikson M. Social phobia in the general population: prevalence and sociodemographic profile. Soc Psychiatry Psychiatr Epidemiol. 1999;34(8):416-24

10. Fuentes-Rodriguez G, Garcia-Lopez L-J, Garcia-Trujillo V. Exploring the role of the DSM-5 performance-only specifier in adolescents with social anxiety disorder. Psychiatry Res. 2018;270:1033-8.

11. Hofmann SG, Albano AM, Heimberg RG, Tracey S, Chorpita BF, Barlow DH. Subtypes of social phobia in adolescents. Depress Anxiety. 1999;9(1):15-8.

12. Hindo CS, González-Prendes AA. One-session exposure treatment for social anxiety with specific fear of public speaking. Res Soc Work Pract. 2011;21(5):528-38.

13. Hofmann SG. Cognitive mediation of treatment change in social phobia. J Consult Clin Psychol. 2004;72(3):392-9.

14. Hofmann SG, Schulz SM, Meuret AE, Moscovitch DA, Suvak M. Sudden gains during therapy of social phobia. J Consult Clin Psychol. 2006;74(4):687-97.

15. Ebrahimi OV, Pallesen S, Kenter RMF, Nordgreen T. Psychological interventions for the fear of public speaking: a meta-analysis. Front Psychol. 2019;10:488.

16. National Institute for Health and Care Excellence. Social anxiety disorder: recognition, assessment and treatment. (NICE Quality Standard No. 159). London: NICE; 2013. https://www.nice.org.uk/guidance/cg159. Accessed 01 July 2019.
17. Öst L-G, Svensson L, Hellström K L indwall R. One-session treatment of specific phobias in youths: a randomized clinical trial. J Consult Clin Psychol. 2001:69(5):814-24.

18. Öst LG, Alm T, Brandberg M, Breitholtz E. One vs five sessions of exposure and five sessions of cognitive therapy in the treatment of claustrophobia. Behav Res Ther. 2001;39(2):167-83.

19. Öst L-G. One-session group treatment of spider phobia. Behav Res Ther. 1996;34(9):707-15.

20. Öst L-G. One-session treatment for specific phobias. Behav Res Ther. 1989;27(1):1-7

21. Lindner P, Miloff A, Hamilton W, Reuterskiöld L, Andersson G, Powers $M B$, et al. Creating state of the art, next-generation Virtual Reality exposure therapies for anxiety disorders using consumer hardware platforms: design considerations and future directions. Cogn Behav Ther. 2017;46(5):404-20.

22. Owens ME, Beidel DC. Can virtual reality effectively elicit distress associated with social anxiety disorder? J Psychopathol Behav Assess. 2015;37(2):296-305.

23. Anderson PL, Price M, Edwards SM, Obasaju MA, Schmertz SK, Zimand E, et al. Virtual reality exposure therapy for social anxiety disorder: a randomized controlled trial. J Consult Clin Psychol. 2013;81(5):751-60.

24. Bouchard S, Dumoulin S, Robillard G, Guitard T, Klinger É, Forget H, et al. Virtual reality compared with in vivo exposure in the treatment of social anxiety disorder: a three-arm randomised controlled trial. Br J Psychiatry. 2018:210(4):276-83.

25. Wallach HS, Safir MP, Bar-Zvi M. Virtual reality cognitive behavior therapy for public speaking anxiety: a randomized clinical trial. Behav Modif. 2009;33(3):314-38.

26. Lindner P, Miloff A, Fagernäs S, Andersen J, Sigeman M, Andersson G, et al Therapist-led and self-led one-session virtual reality exposure therapy for public speaking anxiety with consumer hardware and software: a randomized controlled trial. J Anxiety Disord. 2019;61:45-54.

27. Parrish DE, Oxhandler HK, Duron JF, Swank P, Bordnick P. Feasibility of virtual reality environments for adolescent social anxiety disorder. Res Soc Work Pract. 2016:26(7):825-35.

28. Bartholomay EM, Houlihan DD. Public Speaking Anxiety Scale: preliminary psychometric data and scale validation. Pers Individ Dif. 2016;94:211-5

29. Mattick RP, Clarke JC. Development and validation of measures of socia phobia scrutiny fear and social interaction anxiety. Behav Res Ther. 1998;36(4):455-70.

30. Craske MG, Kircanski K, Zelikowsky M, Mystkowski J, Chowdhury N, Baker A. Optimizing inhibitory learning during exposure therapy. Behav Res Ther. 2008:46(1):5-27.

31. Sripada RK, Rauch SAM. Between-session and within-session habituation in prolonged exposure therapy for posttraumatic stress disorder: a hierarchical linear modeling approach. J Anxiety Disord. 2015;30:81-7.

32. Lee KM. Presence, explicated. Commun Theory. 2004;14(1):27-50.

33. Ling Y, Nefs HT, Morina N, Heynderickx I, Brinkman W-P. A meta-analysis on the relationship between self-reported presence and anxiety in Virtual Reality exposure therapy for anxiety disorders. PLOS ONE. 2014;9(5):96144.

34. Gromer D, Reinke M, Christner I, Pauli P. Causal interactive links between presence and fear in virtual reality height exposure. Front Psychol. 2019;10:141.

35. Laforest $\mathrm{M}$, Bouchard $\mathrm{S}$, Crétu $\mathrm{A}-\mathrm{M}$, Mesly O. Inducing an anxiety response using a contaminated Virtual environment: validation of a therapeutic tool for obsessive-compulsive disorder. Front ICT. 2016;3:18.

36. Hesser $\mathrm{H}$. Modeling individual differences in randomized experiments using growth models: recommendations for design, statistical analysis and reporting of results of internet interventions. Internet Interv. 2015;2(2):110-20

37. Kampmann IL, Emmelkamp PMG, Hartanto D, Brinkman W-P, Zijlstra $\mathrm{BJH}$, Morina N. Exposure to virtual social interactions in the treatment of social anxiety disorder: a randomized controlled trial. Behav Res Ther. 2016;77:147-56.

38. Price $M$, Anderson P. The role of presence in virtual reality exposure therapy. J Anxiety Disord. 2007;21(5):742-51.

39. Miloff A, Lindner P, Dafgård P, Deak S, Garke M, Hamilton W, et al. Automated virtual reality exposure therapy for spider phobia vs. in vivo one-session treatment: a randomized non-inferiority trial. Behav Res Ther 2019;118:130-40. 
40. Baker A, Mystkowski J, Culver N, Yi R, Mortazavi A, Craske MG. Does habituation matter? Emotional processing theory and exposure therapy for acrophobia. Behav Res Ther. 2010;48(11):1139-43.

41. Schwartzman D, Segal R, Drapeau M. Perceptions of virtual reality among therapists who do not apply this technology in clinical practice. Psychol Serv. 2012;9(3):310-5.

42. Segal R, Bhatia M, Drapeau M. Therapists' perception of benefits and costs of using virtual reality treatments. Cyberpsychol Behav Soc Netw. 2011:14(1-2):29-34

\section{Publisher's Note}

Springer Nature remains neutral with regard to jurisdictional claims in published maps and institutional affiliations.
Ready to submit your research? Choose BMC and benefit from:

- fast, convenient online submission

- thorough peer review by experienced researchers in your field

- rapid publication on acceptance

- support for research data, including large and complex data types

- gold Open Access which fosters wider collaboration and increased citations

- maximum visibility for your research: over $100 \mathrm{M}$ website views per year

At BMC, research is always in progress.

Learn more biomedcentral.com/submissions 